\title{
Light-Scattering Polarization Measurements as a New Parameter in Flow Cytometry
}

\author{
B.G. de Grooth, L.W.M.M. Terstappen, G.J. Puppels, and J. Greve \\ Department of Applied Physics, Cell Characterization Group, University of Twente, 7500 AE Enschede, \\ The Netherlands
}

Received for publication August 8, 1986; accepted June 8, 1987

Polarization measurement of orthogonal light scattering is introduced as a new optical parameter in flow cytometry.

In the experimental setup, the electrical field of the incident laser beam is polarized in the direction of the sample flow. The intensity of the orthogonal light scattering polarized along the direction of the incoming laser beam is called depolarized orthogonal light scattering. Theoretical analysis shows that for small values of the detection aperture, the measured depolarization is caused by anisotropic cell structures and multiple scattering processes inside the cell.

Measurements of the orthogonal depolarized light scattering in combination with the normal orthogonal light scattering of human leucocytes revealed two populations of gran- ulocytes. By means of cell sorting it was shown that the granulocytes with a relatively high depolarization are eosinophilic granulocytes. Similar experiments with human lymphocytes revealed a minor subpopulation of yet-unidentified lymphocytes with a relative large orthogonal light-scattering depolarization. The results were obtained with an argon ion laser tuned at different wavelengths as well as with a $630-\mathrm{nm}$ helium neon laser.

These results show that measurement of depolarized orthogonal light scattering is a useful new parameter for flow-cytometric cell differentiation.

Key terms: Eosinophilic granulocytes, neutrophilic granulocytes, depolarization, blood cell differentiation
Light-scattering measurements are routinely used in flow cytometry (FCM). Most frequently, the forward or small angle light scattering $\left(0.5^{\circ}\right.$ to $\left.3^{\circ}\right)$ is detected from which the cell size can be estimated (5). Since the forward light scattering is strongly dependent on the re. fraction index difference between cells and the external medium, cells with a damaged membrane show a decreased forward light scattering. This phenomena is used to discriminate vital from dead cells (3).

Another useful light-scattering parameter is obtained when scattered light within a cone at $90^{\circ}$ with respect to the incoming laser beam is collected. This so-called orthogonal light scattering provides additional information on the structureness of the cells (1). Salzman et al. (6) have shown that human lymphocytes, monocytes, and granulocytes can be distinguished in unstained blood cells by light-scattering measurements, mainly due to differences in orthogonal light scattering. Similar measurements of mouse bone marrow cells, performed by Visser et al,, revealed at least four subpopulations (9). Recently we have shown that human lymphocytes can be divided into two subpopulations on the basis of orthogonal light-scattering measurements $(7,8)$. Cytotoxic lymphocytes, including natural killer cells, show an orthogonal light scattering which is about $70 \%$ larger than that of B-lymphocytes and regulatory T-cells.

Up to now, no efforts have been reported to obtain information on cell morphology by measuring the polarization of light scattering. In this paper we demonstrate that by using this technique a further discrimination between human blood cells can be obtained.

\section{MATERIALS AND METHODS}

Human blood was obtained by venipuncture from healthy individuals. Heparine was used as anticoagulant (150 USP U sodium heparine $/ 10 \mathrm{ml}$ Venoject Terumo Europe NV). Purified lymphocytes and granulocytes were obtained by density separation as described in detail elsewhere (7). Human leucocyte preparations were obtained by adding $190 \mathrm{ml}$ of lysing buffer $(8.29 \mathrm{~g} /$ liter $\mathrm{NH}_{4} \mathrm{Cl}, 0.0037 \mathrm{~g} /$ liter $\mathrm{Na}_{2}$ EDTA, $1.00 \mathrm{~g} /$ liter $\mathrm{KHCO}_{3}$ ) to $10 \mathrm{ml}$ of whole blood and incubating for $20 \mathrm{~min}$ at $4^{\circ} \mathrm{C}$. The lysed blood suspension was washed three times in phosphate-buffered saline (PBS). Cell suspensions were

Address reprint requests to B.G. de Grooth. University of Twente Dept. of Applied Physics, Cell Characterization Group, P,O. Box 217, 7500 AE Enschede, The Netherlands. 
adjusted to a concentration of $1 \times 10^{6} / \mathrm{ml}$ in PBS containing $0.005 \%$ sodium azide and $1 \%$ bovine serum albumin (BSA). Measurements were performed the same day to preserve cell viability.

Monodisperse polystyrene microspheres with a diameter of $1.6 \mu \mathrm{m}$ and standard deviation of $0.05 \mu \mathrm{m}$ were purchased from Polysciences (Polysciences Inc., Warrington, PA; cat. No. 7310).

Flow cytometric experiments were done with a homemade FCM equipped with a $3-\mathrm{W}$ argon ion laser tuned to $488 \mathrm{~nm}$, unless otherwise indicated (Coherent Radiation, Palo Alto, CA: model CR3) or when indicated with a $5 \mathrm{~mW}$ helium neon laser (Spectra Physics, San Jose, $\mathrm{CA}$; model $120 \mathrm{~S}$ ). The optical measurements were performed in a quartz flow cell with a $250 \mu \mathrm{m} \times 250 \mu \mathrm{m}$ square flow channel designed by us and produced by Hellma (Hellma GmbH \& Co, Mullheim/Baden, W. Germany); additional experiments were done with a jet in air, using a FACS flow cell (Becton Dickinson, Sun Valley, CA). The argon laser was focused on the cell stream by means of two cylindrical lenses (focal length 200 and $20 \mathrm{~mm}$ ) and the helium neon laser by means of a single spherical lens (focus length $70 \mathrm{~mm}$ ). The forward light scatter was detected with a photodiode (model Pin 10-D, United Detector Technology) at angles between 2 and $17^{\circ}$. Orthogonal light scattering was collected with a Leitz microscope objective (H32, NA 0.6 collecting angles between $115^{\circ}$ and $65^{\circ}$ ) and imaged on a diaphragm. With a normal glass plate placed at $45^{\circ}$ toward the light beam, a small portion of the scattered light was directed to the first photomultiplier (Hamamatsu R928), which measured the total orthogonal light scattering. The transmitted portion of the light was led to the second photomultiplier (Hamamatsu R928), provided with a polaroid filter (HN7, Melles Griot, Irvine, CA). The filter was placed so as to absorb the scattered light polarized in the direction along the sample stream. The scattered light detected with this photomultiplier is referred to as depolarized orthogonal light scattering.

In order to obtain the absolute values of the depolarization ratio (D), we have calibrated the photomultipliers with respect to each other for both polarization directions. This was done by measuring $I_{1}$ and $I_{\|}$(see theoretical considerations) of monodisperse polystyrene microspheres in two subsequent measurements with one photomultiplier. For these measurements the beam splitter was removed and the polarization filter was placed to transmit $I_{\perp}$ and $I_{\|}$, respectively. The value of D thus obtained was used to calibrate the signals, measured with the two photomultipliers, with respect to each other in the presence of the beam splitter. In this way the polarization effects of the beam splitter are also included.

Data were analyzed with a LSI $11 / 23$ microcomputer and displayed as two-parameter density maps.

\section{THEORETICAL CONSIDERATIONS}

Figure 1 illustrates the optical arrangement of the orthogonal light-scattering experiments described here

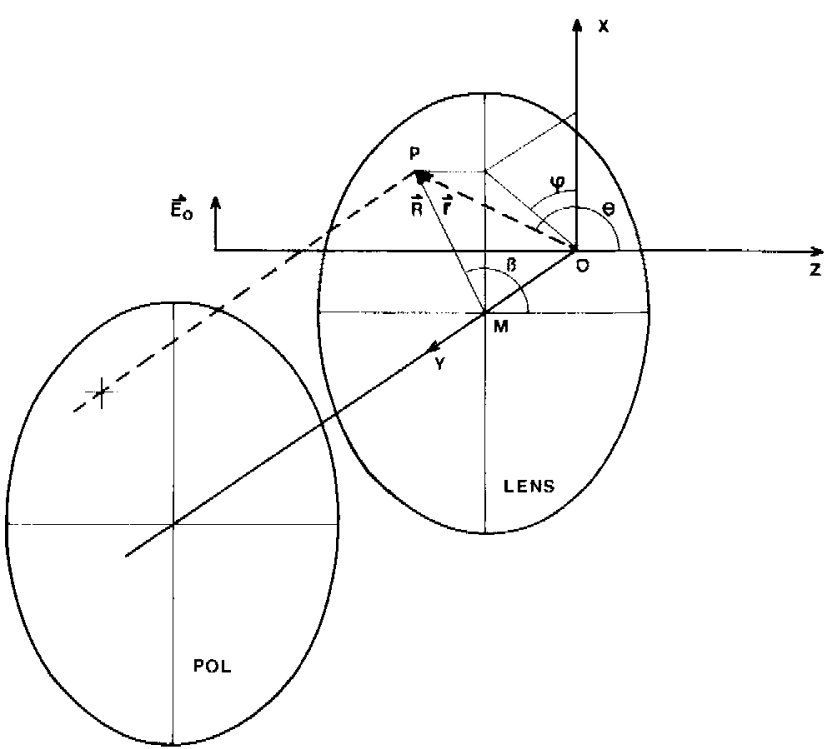

FIG. 1. Schematic drawing of the orthogonal light-scattering experiments defining the geometrical parameters involved. The incident light propagating along the $\mathrm{z}$-axis is polarized along the $\mathrm{x}$-direction. The light is scattered by a particle at the origin 0 . The propagation direction of the scattered light is described by polar coordinates $r, \theta$, and $\phi$. The light is collected with a lens placed at the focal distance from the origin and analyzed with a polarizer $(\mathbf{P})$, See Appendix for derivations of formal equations

and defines the geometrical parameters used. An incom. ing laser beam propagates along the $\mathrm{z}$-axis and is linearly polarized with incident electrical field $\mathrm{E}_{0}$ along the $\mathrm{x}$-axis. At the origin of our coordinate system the laser beam intersects the particles of the flow cytometer moving along the $\mathrm{x}$-axis. The direction of the scattered light is described in spherical coordinates $r, \theta$, and $\phi$.

In general the amplitudes of the scattered light polarized in the $\theta$ and $\phi$ direction can now be described as (2)

$$
\begin{aligned}
& E_{\phi}=\left(S_{4} \cos \phi-S_{1} \sin \phi\right) f E_{0} \\
& E_{\theta}=\left(S_{2} \cos \phi-S_{3} \sin \phi\right) f E_{0}
\end{aligned}
$$

where $f$ is a complex expression for a spherical wave, inversely proportional to $r$, the distance between the scatterer and the detector, and $S_{1}, S_{2}, S_{3}$, and $S_{4}$ are the so-called amplitude functions (2), dependent on $\phi, \theta$, and the geometry and physical composition of the scatterer. The measurable light-scattering intensities $\mathrm{I}_{\dot{\phi}}$ and $\mathrm{I}_{t}$ are related to these fields by

$$
\begin{aligned}
& I_{\phi}=\left|E_{\phi}\right|^{2} \\
& I_{\theta}=\left|E_{\phi}\right|^{2}
\end{aligned}
$$

The light intensities measured using ideal polarizers transmitting only the $\mathrm{x}$ polarized or the $\mathrm{z}$ polarized fields are called $I_{\|}$and $I_{\perp}$, respectively. $I_{\perp}$ is called the depolarized light-scattering intensity and the depolarization ratio is defined here as 


$$
\mathrm{D}=\frac{\mathrm{I}_{\perp}}{\mathrm{I}_{\perp}+\mathrm{I}_{\|}}
$$

For light scattered in the yz plane, i.e., orthogonal with respect to the polarization direction of the incident light, we have $\phi=90^{\circ}$ and we can write for $I_{\|}$and $I_{\perp}$ :

$$
\begin{aligned}
& I_{\|}=\left|S_{1} f E_{0}\right|^{2} \\
& I_{\perp}=\left|S_{3} f E_{0}\right|^{2}
\end{aligned}
$$

Thus, whereas $I_{\|}$is determined by $S_{1}$, measurement of the depolarized light-scattering intensity in this geometry yields information on the term $S_{3}$. The importance of this term will be illustrated briefly.

Mie theory shows that for homogeneous spheres, spherical shells, etc., the term $S_{3}$ is zero (2). $S_{3}$ becomes important for particles which are optically anisotropic (due to shape and/or composition) and for particles containing structures, so that multiple scattering becomes important. A simple but striking example of the latter is illustrated in Figure 2. We imagine a cell containing two intracellular spheres located on the $\mathrm{x}$-axis. A particular incoming ray, traveling along the z-axis, is reflected along the $\mathrm{x}$-axis by the first sphere and along the $\mathrm{y}$ axis by the second sphere. Applying the laws of reflection we find that the polarization direction of this ray changes from along the $\mathrm{x}$-axis to along the $\mathrm{z}$-axis. Thus we see how multiple reflections can lead to depolarization and thus to contributions to the term $S_{3}$. The reader can easily verify this example with two mirrors and two polarizer sheets. In a similar way it can be shown that multiple scattering due to diffraction and refraction can also lead to depolarization (Puppels, unpublished results).

In a practical flow cytometer, the optical configuration is such that the detector, which collects orthogonally scattered light, gathers light from a conus around the $y$ axis. In that case depolarization is not only caused by $\mathrm{S}_{3}$ but also by the other amplitude functions $\mathrm{S}_{1}, \mathrm{~S}_{2}$, and $\mathrm{S}_{4}$.

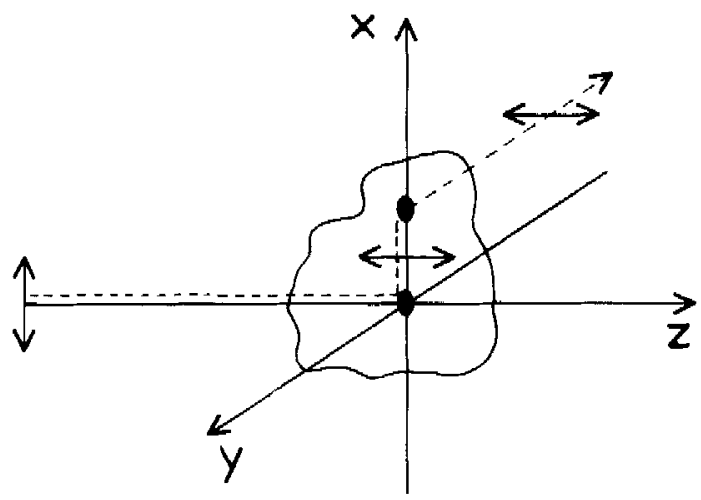

FIG. 2. Depolarization of orthogonal light scattering by multiplescattering processes. An incident light ray is traced as it is reflected by two intracellular particles. The final scattered ray propagating along the negative $y$-axis is completely polarized in the $z$-direction.
This extra depolarization is due to the geometry of the measuring system and is called cross depolarization. It is closely related to the term "aperture polarization" used when describing fluorescence polarization experiments. In order to determine the origin of a measured depolarization signal we can analyze the dependence of the depolarization ratio on the angle $\phi$. We place a rectangular diaphragm in front of the collection lens, limiting the angles of the collected light between $\phi=90^{\circ}$ $\pm \Delta \phi$ and $\theta=90^{\circ} \pm \Delta \theta$. As is shown in the appendix, depolarization due to pure cross depolarization drops to zero proportional to $\Delta \phi^{2}$. The polarization ratio due to the $S_{3}$ term, however, reaches a constant nonzero value for small values of $\Delta \phi$.

\section{RESULTS}

We have investigated the orthogonally depolarized light scattering of human blood cells measured with a flow cytometer. Lymphocytes, granulocytes, and monocytes were selected by gating on forward and orthogonal light scattering of lysed peripheral blood cell preparations obtained from healthy donors. Erythrocytes were taken from whole blood diluted in PBS.

In Figure 3 two-parameter density maps of the depolarized orthogonal light scattering vs. the total orthogonal light scattering are plotted for the different cell populations. Figure 3a clearly reveals two populations of granulocytes. From Figure $3 b$ it appears that the large majority of the lymphocytes and all monocytes have about the same polarization ratio (depolarized light scattering divided by the total orthogonal light scattering) but that a minor subpopulation of lymphocytes exists with a relatively large depolarization. The monocytes with a relatively larger orthogonal light scattering show no heterogenity (Fig. 3b). The results of Figure $3 \mathrm{a}$ and $\mathrm{b}$ could be reproduced with granulocytes and lymphocytes purified with density separation. Erythrocytes show no heterogenity (Fig. 3c).

The granulocyte subpopulations have been sorted and examined under the light microscope after May-Grünwald staining. The cells with a relatively high depolarization of orthogonal light scattering were identified as eosinophylic granulocytes (99\% purity) whereas the other population consists of neutrophilic granulocytes (99\% purity). The identification of the minor lymphocyte subpopulation of Figure $3 \mathrm{~b}$ is still under investigation.

Whether the measured depolarization signals are due to cross depolarization or due to cellular structures was examined according to the method outlined in the theoretical considerations given above. The measured depolarization ratio was plotted vs. the acceptance angle $\Delta \phi$ of the collection objective for the different cell populations (Fig. 4). The curves were normalized at the maximum angle of $14.5^{\circ}$. Also included are the values measured with polystyrene microspheres with a diameter of $1.16 \mu \mathrm{m}$ and a theoretical curve for perfect homogeneous spheres.

The absolute values of the depolarization ratios of the different particles are given in Table 1 for a small and a 

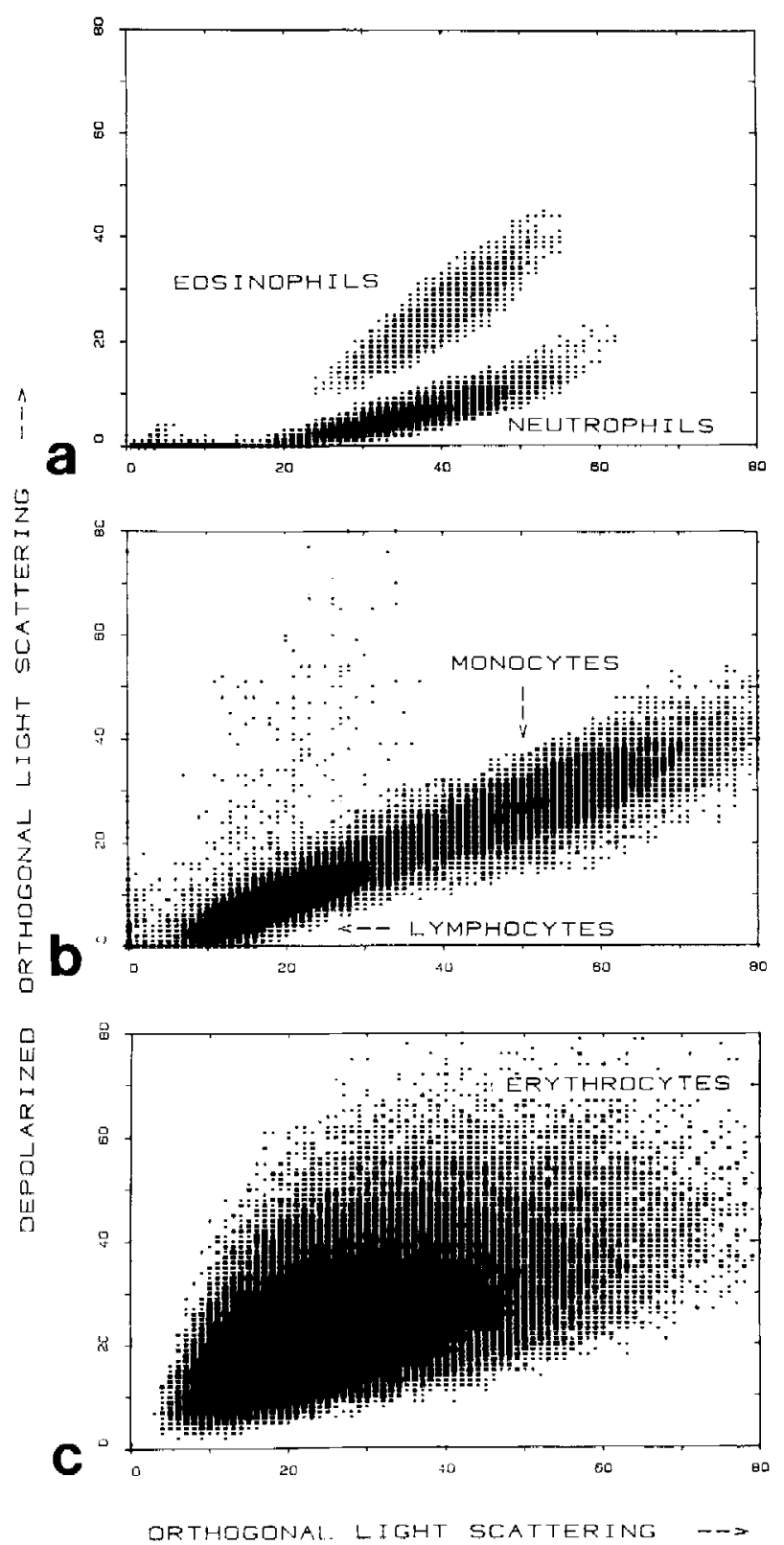

FIG. 3. Flow cytometric density map of orthogonal light scattering vs. depolarized orthogonal light scattering of different human blood cells, a) Lysed peripheral blood revealing neutrophilic and eosinophilic granulocytes. b) The same sample as Figure 3a but at different amplifications showing lymphocytes and monocytes. c) Erythrocytes.

large value of the angle of acceptance $\Delta \phi$.

Since the intensity of the depolarized orthogonal light scattering is not very high we verified that the measured light is indeed only due to elastic light scattering and not caused by autofluorescence. This was done by inserting a 500-nm shortwave pass filter in the detection optic; it did not affect the results. In addition, the illumination wavelength of the argon ion laser used was

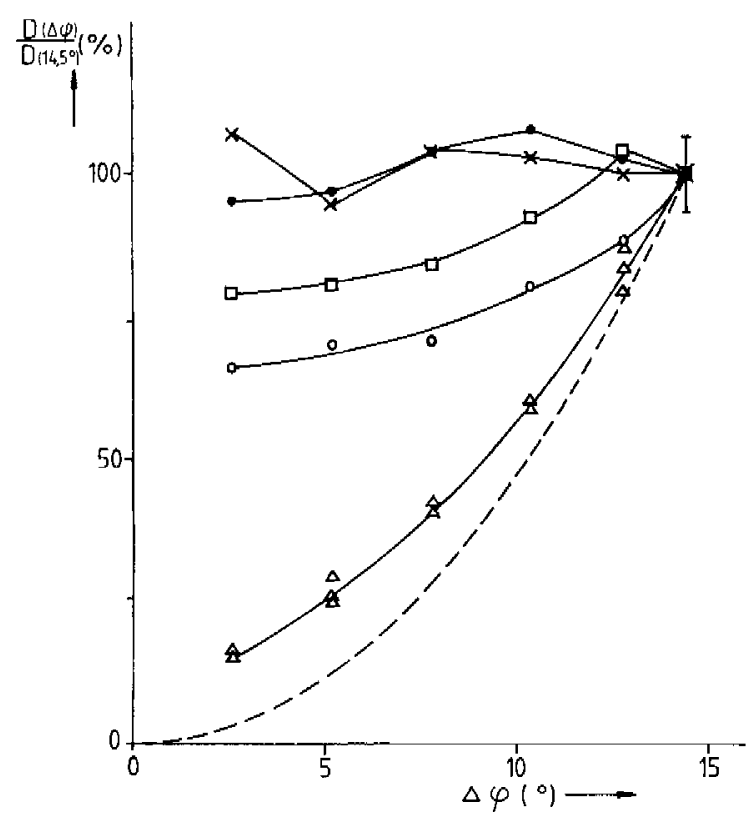

Fic. 4. Normalized depolarization ratio measured as function of the acceptance angle $\Delta \phi$ for neutrophils $(\bullet)$, eosinophils $(\times)$, lymphocytes $(\bigcirc)$, monocytes ( $\square$ ), and microspheres with a diameter of $1.16 \mu \mathrm{m}(\Delta)$. The dashed line represents the theoretical curve for ideal spheres. The curves were normalized at a value for $\Delta \phi$ of $14.5^{\circ}$.

changed from 488 to 509 and $514 \mathrm{~nm}$, and a helium neon laser of $630 \mathrm{~nm}$ was used. In all cases, the results obtained were the same.

Many flow cytometers, in particular flow sorters, make use of a jet in air configuration. In these systems a small obscuration bar is placed in front of the orthogonal light detection objective in order to block the laser light scattered by the cylindrical liquid jet. With such an obscuration bar, the relative contribution of the cross depolarization to the total depolarization is increased. We have used such a system with an obscuration bar effectively blocking the orthogonal light scattering with an angle $\phi$ between $87^{\circ}$ and $92^{\circ}$. With this system using a $5 \mathrm{~mW}$ helium neon laser as light source, a clear discrimination between neutrophilic and eosinophilic granulocytes still was obtained (Fig. 5).

Table 1

Absolute Values of the Depolarization Ratios $(D)^{\mathrm{a}}$

\begin{tabular}{llc}
\hline & $\Delta \phi=2.5^{\circ}$ & $\Delta \phi=14.5^{\circ}$ \\
\hline $\begin{array}{l}\text { Eosinophilic } \\
\text { granulocytes }\end{array}$ & 0.047 & 0.044 \\
Neutrophilic & 0.013 & 0.013 \\
granulocytes & 0.005 & \\
Monocytes & 0.005 & 0.007 \\
Lymphocytes & 0.0062 & 0.008 \\
Microspheres & & 0.039 \\
\hline
\end{tabular}

${ }^{2} \mathrm{D}=\mathrm{I}_{\perp} /\left(\mathrm{I}_{\perp}+\mathrm{I}_{\|}\right)$is given for a small $\left(\Delta \phi=2.5^{\circ}\right)$ and a large $\left(\Delta \phi=14.5^{\circ}\right)$ value of the angle of acceptance $\Delta \phi$ of eosinophilic, neutrophilic granulocytes, monocytes, lymphocytes, and polystyrene microspheres with a diameter of $1.16 \mu \mathrm{m}$. 


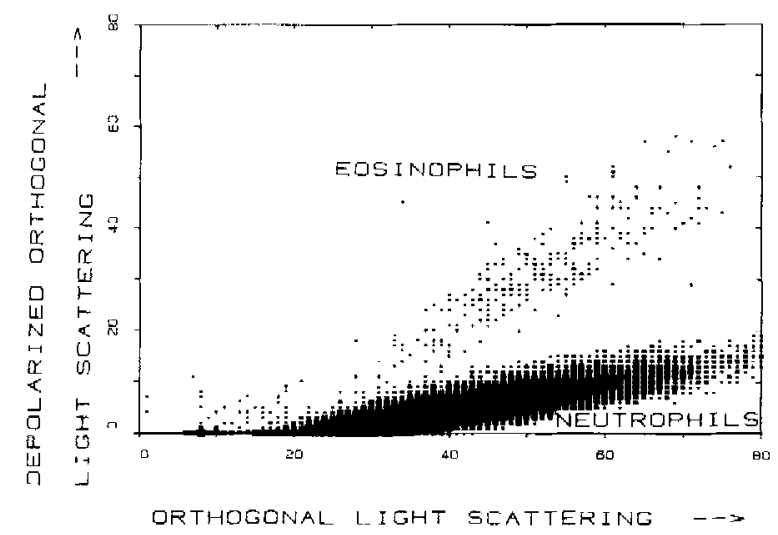

FIG. 5. Density map of orthogonal light scattering vs. the depolarized orthogonal light scattering of lysed whole blood measured with a $5 \mathrm{~mW}$ helium neon laser and a stream in air flow system. A clear discrimination between eosinophilic and neutrophilic granulocytes is obtained.

\section{DISCUSSION}

We have shown that measurement of the depolarized orthogonal light scattering adds to existing flow cytometers a new parameter which can provide useful information not available by other means. The observation that, with this parameter, eosinophylic granulocytes can be distinguished from neutrophylic granulocytes, is of practical as well as of theoretical importance. It is now feasible to construct a simple flow cytometer, provided with a low-power helium neon laser, which can perform white blood cell differentiation with unstained cells. The cells can be differentiated into cytotoxic lymphocytes (8), noncytotoxic lymphocytes (8), monocytes (6), neutrophilic granulocytes, and eosinophilic granulocytes (this study). Up to now, additional staining procedures had to be applied, or the weak autofluorescence had to be measured with an expensive argon ion laser (10). From a theoretical point of view, it is interesting to note that the eosinophils, with a large number of small intracellular particles (granulae), show a relative high depolarization ratio. This is in agreement with theoretical considerations that multiple-scattering processes result in depolarization. In Figure 4 we have investigated the nature of the measured depolarization signal. As discussed in the theoretical section, the cross-depolarization contributions vanish proportional to $\Delta \phi^{2}$. From the figure we may conclude that the observed depolarization of both granulocyte populations is mainly due to true cellular depolarization effects. For lymphocytes and monocytes about $25 \%$ of the depolarization measured with the full aperture (na $=0.6$ ) might be due to cross depolarization.

The observed angle dependency of the depolarization ratio found with microspheres does not completely fol. low the theoretically expected behaviour for ideal spheres. For small values of $\Delta \phi$ a constant depolariza- tion ratio is reached. This can either be caused by imperfect optical components or geometry used in the measurements or by imperfections in the spheres. The minor subpopulation of Figure $3 \mathrm{~b}$ with a large depolarization could be cytotoxic cells in view of the relatively large orthogonal light scattering.

Weil and Chused (10) have shown that eosinophilic granulocytes show a larger autofluorescence than neutrophilic granulocytes. By changing the illumination and detection wavelengths we have excluded that autofluorescence contributes significantly to our depolarization measurements.

The frequently used jet in air flow cytometers are not particularly well suited for depolarization experiments since the angular region with minimal contributions from cross depolarization is blocked. However, even with such a system we could clearly resolve the two granulocyte subpopulations. This is in agreement with the results given in Figure 4 which show that cross depolarization, even for large apertures, is small for granulocytes.

We have shown that the explicit use of the polarization of the orthogonal light scattering extends the ability of flow cytometric cell differentiation. Measurement of depolarized light scattering can also be applied to other angles, e.g., forward and/or backward light scattering. We are currently investigating in our laboratory whether such measurements give additional information.

\section{LITERATURE CITED}

1. Benson MC, MeDougal DC, Coffey DS: The applications of perpendicular and forward light scatter to assess nuclear and cellular morphology. Cytometry 5:515-522, 1984.

2. Hulst van de HC: Light scattering by small particles. Dover Publications, Inc., New York, 1981, Chapter 4, pp 28-39.

3. Julius MH, Sweet RG, Fatham CG: Fluorescence-activated cell sorting and its applications. In: Mammalian Cells: Probes and Probloms, ERDA Symposium Series CONF-731007, Richmond CR, Petersen DF, Mullaney PF, et al. (eds). Technical Information Center, Oak Ridge, Tenn., 1975, p 107.

4. Marston PL: Uniform Mie theoretic analysis of polarized and crowpolarized optical glories. J Opt Soc Am 73:1816-1819, 1983.

5. Mullaney PF, Dean PN: The small angle light scattering of biological cells. Biophys $J$ 10:764-772, 1970.

6. Salzman GC, Growell JM, Martin JC: Cell classification by laser light scattering: Identification and separation of unslained leukocytes. Acta Cytol (Baltimore) 19:374-377, 1975.

7. Terstappen I.WMM, de Grooth BG, Nolten GMJ, ten Napel CHH, van Berkel W, Greve J: Physical discrimination between human T-lymphocyte subpopulations by means of light scattering, revealing two populations of T8-positive cells. Cytometry 7:178-183, 1986.

8. Terstappen LWMM, de Grooth BG, ten Napel CHH, van Berkel W, Greve J: Discrimination of human cytotoxic lymphocytes from regulatory and B-lymphocytes by orthogonal light scattering. $J$ Immunol Methods 95:211-216, 1986.

9. Visser JW, Van den Engh GJ, van Bekkum DW: Light scattering properties of murine hematopoietic cells. Blood Cells 6:391-407, 1980.

10. Weil GJ, Chused TM: Eosinophil autofluorescence and its use in isolation and analysis of human eosinophils using flow microfluorometry. Biood 57:1099, 1981 . 


\section{APPENDIX}

In this appendix we will derive the formal equations for the amplitudes of the scattered electrical fields, as collected with an ideal lens and analyzed with a polaroid sheet. We will use the notation of Marston (4). Figure 1 shows the basic geometry. The lens is centered around the $y$-axis at point $M$ and placed parallel to the $x z$ plane at the focal distance from the origin 0 . When the scattered wave reaches the lens it can be described by

$$
\overrightarrow{\mathrm{E}}_{\mathrm{sca}}=\mathrm{E}_{\theta} \overrightarrow{\mathrm{e}}_{\theta}+\mathrm{E}_{\phi} \overrightarrow{\mathrm{e}}_{\phi}
$$

with $\mathrm{E}_{\theta}$ and $\mathrm{E}_{\phi}$ given by eq. 1 and 2 .

The scattered wave enters the lens at point $P$. We define the vector $R$ from $M$ to $P$ and the angle between $\mathbf{R}$ and the z-axis $\beta$. The unit vector $\overrightarrow{\mathrm{e}}_{\beta}$, defined in the usual way, is perpendicular to $e_{r}$ and thus lies in the plane through $\overrightarrow{\mathrm{e}}_{\phi}$ and $\overrightarrow{\mathrm{e}}_{\theta}$. Therefore we can rewrite the previous equation as

$$
\overrightarrow{\mathrm{E}}_{\mathrm{sca}}=\mathrm{E}_{\beta} \overrightarrow{\mathrm{e}}_{\rho}+\mathrm{E}_{\rho} \overrightarrow{\mathrm{e}}_{\rho}
$$

where $\vec{e}_{\theta}$ is in the plane through $\vec{e}_{\phi}$ and $\vec{e}_{\theta}$ and is orthogonal to $\overrightarrow{\mathrm{e}}_{\beta}$. $\mathrm{E}_{\beta}$ and $\mathrm{E}_{\rho}$ can be obtained by calculating

$$
\begin{aligned}
& \mathrm{E}_{\beta}=\left(\overrightarrow{\mathrm{e}}_{\theta} \cdot \overrightarrow{\mathrm{e}}_{\beta}\right) \mathrm{E}_{\theta}+\left(\overrightarrow{\mathrm{e}}_{\phi} \cdot \overrightarrow{\mathrm{e}}_{\beta}\right) \mathrm{E}_{\phi} \\
& \mathrm{E}_{\rho}=\left(\overrightarrow{\mathrm{e}}_{\theta} \cdot \overrightarrow{\mathrm{e}}_{\rho}\right) \mathrm{E}_{\theta}+\left(\overrightarrow{\mathrm{e}}_{\phi} \cdot \overrightarrow{\mathrm{e}}_{\rho}\right) \mathrm{E}_{\phi}
\end{aligned}
$$

After the scattered field has passed the lens, the propagation direction is parallel toward the $y$-axis and the amplitude can be expressed in the polar coordinates $\overrightarrow{\mathrm{e}}_{\mathrm{R}}$ and $\overrightarrow{\mathrm{e}}_{\beta}$

$$
\overrightarrow{\mathrm{E}}_{\mathrm{sca}}=\mathrm{E}_{\mathrm{R}} \overrightarrow{\mathrm{e}}_{\mathrm{R}}+\mathrm{E}_{\beta} \overrightarrow{\mathrm{e}}_{\beta}
$$

By passing through the lens, the amplitude in the direction of $\mathrm{e}_{\beta}$ remains unchanged, whereas $\mathrm{E}_{\mathrm{R}}=\mathrm{E}_{\rho}$. If we now analyze the scattered wave in the direction parallel and perpendicular to the polarization direction of the incident field, with $e_{\|}=e_{x}$ and $e_{\perp}=e_{z}$, we obtain:

$$
\overrightarrow{\mathrm{E}}_{\mathrm{sca}}=\mathrm{E}_{\|} \overrightarrow{\mathrm{e}}_{\|}+\mathrm{E}_{\perp} \overrightarrow{\mathbf{e}}_{\perp}
$$

with

$$
\begin{aligned}
\mathrm{E}_{\|} & =\left[\mathrm{E}_{\theta}\left(\overrightarrow{\mathrm{e}}_{\theta} \cdot \overrightarrow{\mathrm{e}}_{\rho}\right)+\mathrm{E}_{\phi}\left(\overrightarrow{\mathrm{e}}_{\phi} \cdot \overrightarrow{\mathrm{e}}_{\rho}\right)\right]\left(\overrightarrow{\mathrm{e}}_{\mathbf{R}} \cdot \overrightarrow{\mathrm{e}}_{\mathrm{x}}\right) \\
& +\left[\mathrm{E}_{\theta}\left(\overrightarrow{\mathrm{e}}_{\theta} \cdot \overrightarrow{\mathrm{e}}_{\beta}\right)+\mathrm{E}_{\rho}\left(\overrightarrow{\mathrm{e}}_{\phi} \cdot \overrightarrow{\mathrm{e}}_{\beta}\right)\right]\left(\overrightarrow{\mathrm{e}}_{\beta} \cdot \overrightarrow{\mathrm{e}}_{\mathrm{x}}\right)
\end{aligned}
$$

$$
\begin{aligned}
\mathrm{E}_{\perp} & =\left[\mathrm{E}_{\theta}\left(\overrightarrow{\mathrm{e}}_{\theta} \cdot \overrightarrow{\mathrm{e}}_{\phi}\right)+\mathrm{E}_{\phi}\left(\overrightarrow{\mathrm{e}}_{\phi} \cdot \overrightarrow{\mathrm{e}}_{\phi}\right)\right]\left(\overrightarrow{\mathrm{e}}_{\mathrm{R}} \cdot \overrightarrow{\mathrm{e}}_{z}\right) \\
& +\left[\mathrm{E}_{\theta}\left(\overrightarrow{\mathrm{e}}_{\theta} \cdot \overrightarrow{\mathrm{e}}_{\beta}\right)+\mathrm{E}_{\phi}\left(\overrightarrow{\mathrm{e}}_{\phi} \cdot \overrightarrow{\mathrm{e}}_{\beta}\right)\right]\left(\overrightarrow{\mathrm{e}}_{\beta} \cdot \overrightarrow{\mathrm{e}}_{z}\right)
\end{aligned}
$$

By geometrical analysis the values of the scalar products can be obtained and in combination with 1 and 2 we get

$$
\begin{aligned}
& \mathrm{E}_{\|}=\frac{(\sin \phi+\sin \theta)\left(\mathrm{S}_{4} \cos \phi-\mathbf{S}_{1} \sin \phi\right)-\cos \phi \cos \theta\left(\mathrm{S}_{2} \cos \phi-\mathrm{S}_{3} \sin \phi\right)}{1+\sin \phi \sin \theta} \cdot \text { f } \mathbf{E}_{0} \\
& \mathrm{E}_{\perp}=\frac{(\sin \phi+\sin \theta)\left(\mathrm{S}_{2} \cos \phi-\mathbf{S}_{3} \sin \phi\right)-\cos \phi \cos \theta\left(\mathbf{S}_{4} \cos \phi-\mathbf{S}_{1} \sin \phi\right)}{1+\sin \phi \sin \theta} \cdot \text { f } \mathbf{E}_{0}
\end{aligned}
$$

We see that for a practical situation $\mathrm{E}$ is not only dependent on $S_{3}$ but also on the other amplitude functions $S_{1}$, $\mathrm{S}_{2}$, and $\mathrm{S}_{4}$. These latter contributions are called cross or aperture depolarization.

We take $\phi=90^{\circ}+\Delta \phi$ and $\theta=90^{\circ}+\Delta \theta$. For small values of $\Delta \phi$ and $\Delta \theta$ we get

$$
\mathrm{E}_{1}=\left[-\mathrm{S}_{3}+\Delta \phi \mathrm{S}_{2}+\mathrm{O}\left(\Delta \phi^{2}, \Delta \theta^{2}, \Delta \phi \Delta \theta\right)\right] \cdot f \mathrm{E}_{0}
$$

where $\mathrm{O}\left(\Delta \phi^{2}, \Delta \theta^{2}, \Delta \phi \Delta \theta\right)$ stands for higher orders than $\Delta \phi$ and $\Delta \theta$. Thus, the depolarized orthogonal light scattering is determined by $S_{3}$ and the cross depolarization is in first-order approximation dependent on $\Delta \phi \mathrm{S}_{2}$. To analyze the $\Delta \phi$ dependency we maintain a fixed value for $\Delta \theta$ and vary $\Delta \phi$. If the depolarization is entirely due to cross depolarization we have $S_{3}=S_{4}=O$ (as for ideal spheres) and we obtain

$$
\begin{aligned}
& \mathbf{R}=\frac{\mathbf{I}_{\perp}}{\mathbf{I}_{\|}+\mathbf{I}_{1}} \\
& =\frac{\int_{90-\Delta \phi}^{90+\Delta \phi}}{90+\Delta \phi} \int_{90-\Delta \theta}^{90+\Delta \theta}\left|E_{\perp}\right|^{2} \mathrm{~d} \theta \mathrm{d} \phi \\
& \int_{90-\Delta \omega} \int_{90-\Delta \theta}\left|E_{l}\right|^{2}+\left|E_{\perp}\right|^{2} \mathrm{~d} \theta \mathrm{d} \phi \\
& =\frac{1}{3} \triangle \phi^{2} \cdot \mathrm{k}
\end{aligned}
$$

where $\mathrm{k}$ is independent on $\Delta \phi$. The polarization term due to $S_{3}$, is independent of $\Delta \phi$. 